\title{
Developmental origins of health and disease (DOHaD)
}

\author{
Origens desenvolvimentistas da saúde e da doença (DOHaD) \\ Patrícia P. Silveira ${ }^{1}$, André K. Portella ${ }^{2}$, Marcelo Z. Goldani ${ }^{3}$, Marco A. Barbieri ${ }^{4}$
}

\section{Resumo}

Objetivo: Apresentar um novo ramo da ciência, denominado origens desenvolvimentistas da saúde e doença (DOHaD), abordando conceitos, métodos de estudo, aspectos éticos e perspectivas para essa área do conhecimento.

Fontes dos dados: Revisão não sistemática da literatura biomédica, com o intuito de obter referências históricas e atualizadas relacionadas com o tema em discussão.

Síntese dos dados: Estudos recentes demonstram associações entre agravos ocorridos em fases iniciais do desenvolvimento somático e a amplificação do risco para doenças crônicas ao longo da vida, tais como obesidade, diabetes e doenças cardiovasculares. Diferentes modelos foram propostos na tentativa de melhor explicar essas associações, como a teoria do fenótipo poupador, a programação, as respostas adaptativas preditivas e o conceito de concordância ou contraste. Alguns dos possíveis mecanismos envolvidos nesses processos são: efeitos do ambiente sobre a expressão gênica, através de mecanismos epigenéticos; efeitos de sinais hormonais transmitidos ao feto através da placenta ou ao recém-nascido através da lactação.

Conclusões: O DOHaD agrega informações advindas de várias áreas do conhecimento, propondo novas metodologias de investigação no sentido de esclarecer a influência de eventos adversos ocorridos em fases precoces do desenvolvimento humano sobre o padrão de saúde e doença ao longo da vida. Esse novo campo da ciência propõe novos modelos de causalidade e mecanismos envolvidos no surgimento e desenvolvimento de doenças crônicas. Os resultados dessas investigações poderão resultar em impacto significativo na prevenção de doenças crônicas, bem como na promoção de saúde em diferentes fases da vida.

J Pediatr (Rio J). 2007;83(6):494-504: DOHaD, programação, hipótese de Barker.

\section{Introdução}

Estudos epidemiológicos em diferentes partes do mundo relacionam a influência de determinados fatores ambientais

\begin{abstract}
Objective: To present a new branch of scientific knowledge, known as the developmental origins of health and disease (DOHaD), covering its concepts, study methods and ethical considerations in addition to the prospects for this area of knowledge.
\end{abstract}

Sources: A non-systematic review of the biomedical literature intended to identify historical and current references related to the subject under discussion.

Summary of the findings: Recent studies demonstrate associations between aggressions suffered during the initial phases of somatic development and amplified risk of chronic diseases throughout life, such as obesity, diabetes and cardiovascular diseases. A variety of models have been proposed in attempts to better explain these associations, such as the thrifty phenotype, programming and predictive adaptive response theories and the concept of match or mismatch. Some of the mechanisms possibly involved in these processes are: effects of the environment on gene expression, through epigenetic mechanisms; effects of hormonal signals transmitted to the fetus via the placenta or the newborn via lactation.

Conclusions: DOHaD draws together information originating from many different areas of knowledge, proposing new investigative methodologies to elucidate the influence of adverse events that occur during early phases of human development on the pattern of health and disease throughout life. This new scientific field proposes new models of causality and of the mechanisms involved in the emergence and development of chronic diseases. The results of these investigations may result in a significant impact on the prevention of chronic diseases, and also on health promotion in different phases of life.

J Pediatr (Rio J). 2007;83(6):494-504: DOHaD, programming, Barker hypothesis.

do início da vida com alterações na expressão da carga genética do indivíduo, determinando um padrão de saúde-doença peculiar. Igualmente, estudos clínicos e pré-clínicos apontam para a mesma direção, sugerindo uma forte associação entre

1. Pediatra. Doutora, Universidade Federal do Rio Grande do Sul (UFRGS), Porto Alegre, RS.

2. Pediatra. Mestre, UFRGS, Porto Alegre, RS.

3. Pediatra. Doutor, Faculdade de Medicina de Ribeirão Preto, Universidade de São Paulo (USP), Ribeirão Preto, SP. Professor, Departamento de Pediatria e Puericultura, UFRGS, Porto Alegre, RS.

4. Pediatra. Professor titular, Departamento de Puericultura e Pediatria, Faculdade de Medicina de Ribeirão Preto, USP, Ribeirão Preto, SP.

Não foram declarados conflitos de interesse associados à publicação deste artigo.

Como citar este artigo: Silveira PP, Portella AK, Goldani MZ, Barbieri MA. Developmental origins of health and disease (DOHaD). J Pediatr (Rio J). 2007; 83(6):494-504.

Artigo submetido em 18.07.07, aceito em 13.08.07.

doi 10.2223/JPED.1728 
agravos ambientais ocorridos na vida fetal ou nas fases iniciais da vida extra-uterina e o surgimento de doenças crônicas ao longo da vida. Esses achados apontam para novas pontes de causalidade, inferindo a possibilidade de construção precoce de ajustes metabólicos determinantes de desfechos mórbidos ao longo da vida. Neste artigo de revisão, temos como objetivo abordar essas idéias, atualmente agrupadas em um novo ramo de conhecimento científico sob a nomenclatura de origens desenvolvimentistas da saúde e doença (DOHaD).

\section{Contexto histórico}

Com a redução de taxas de mortalidade entre os recémnascidos (RN) pré-termo em estágios do desenvolvimento muito precoces, novas doenças emergiram e foram identificadas. Por exemplo, Avery \& Mead, em 1959, descreveram pela primeira vez a doença da membrana hialina, caracterizada pela imaturidade pulmonar e incapacidade de produção de surfactante ${ }^{1}$. Outro exemplo é a enterocolite necrosante, relacionada à imaturidade intestinal ${ }^{2,3}$. Ainda outras entidades de caráter mais crônico, como a displasia broncopulmonar $^{4}$ e a retinopatia da prematuridade ${ }^{5}$, surgiram como resultado do desenvolvimento de terapias agressivas para suporte dessa população especial.

Não foi difícil supor que o emprego de novas tecnologias utilizadas para ampliar a possibilidade de sobrevida de RN prétermo ou com baixo peso ao nascer acometidos por essas novas doenças pudessem produzir conseqüências a longo prazo. Porém, mais distante ainda seria correlacionar situações supostamente fisiológicas e adaptativas do organismo materno, fetal ou do RN diante de condições ambientais adversas com desfechos futuros. Em meados da década de 1930 do século passado, enquanto estudavam as taxas de mortalidade na Inglaterra e na Suécia, pesquisadores surpreenderam-se com a constatação de que as condições ambientais durante a vida fetal e a infância pareciam estar determinando a sobrevida de cada geração ${ }^{6}$.

Na década de 1970, Ravelli et al. estudaram uma população de 300.000 homens, filhos de mulheres expostas a um período de escassez alimentar (fome holandesa), durante o cerco da Holanda pela Alemanha na Segunda Guerra Mundial. Na vida adulta, esses indivíduos apresentaram padrões diferenciados de composição corporal dependendo da idade em que tinham sido expostos à desnutrição materna durante a vida intra-uterina. Caso a mãe houvesse sofrido desnutrição durante o último trimestre da gestação, esse grupo apresentava uma baixa incidência de obesidade. No entanto, se a desnutrição ocorresse no primeiro semestre da gestação, a incidência de obesidade aumentava significativamente na prole $^{7}$.

Nos anos 1960, Neel lançou a "hipótese do genótipo poupador ${ }^{\prime \prime}$, propondo que determinadas populações apresentavam maior propensão à resistência insulínica, tanto por seleção quanto por aspectos genéticos. Segundo esse autor, uma mutação aleatória levando à resistência à insulina poderia ser adaptativa e benéfica para indivíduos expostos a ambientes com escassez alimentar, originando seleção natural desses indivíduos, com a conseqüente transmissão do caráter para as gerações seguintes. No entanto, evidências atuais sobre interações entre gene e ambiente demonstram que considerações sobre efeitos puramente genéticos e independentes do meio não têm um correlato biológico verdadeiro.

\section{Modelos causais}

Seguindo esse raciocínio, Barker et al. desenvolveram a hipótese de que condições adversas intra-uterinas e durante a infância aumentavam o risco de doenças cardiovasculares. Para testá-la, correlacionaram o peso ao nascer e as condições ambientais durante a infância com a saúde cardiovascular de indivíduos adultos nascidos no início do século XX, em Hertfordshire, Inglaterra ${ }^{9}$. Nesses estudos, demonstraram que pessoas nascidas com baixo peso permaneceram biologicamente diferentes daquelas com peso adequado, de forma persistente, até a vida adulta. Elas apresentavam maior pressão arterial ${ }^{10}$, sendo mais propensas a desenvolver diabetes tipo $2^{11}$. Além disso, em achados subseqüentes, esses e outros pesquisadores demonstraram que o baixo peso ao nascer se associava a um padrão alterado de lipídeos plasmáti$\cos ^{12}$, redução da densidade óssea ${ }^{13}$, respostas ao estresse diferenciadas ${ }^{14}$, artérias menos elásticas ${ }^{15}$, padrões de secreção hormonal específicos ${ }^{16,17}$ e maior incidência de depressão ${ }^{18,19}$. Essas observações geraram a "hipótese do fenótipo poupador" (thrifty phenotype hypothesis), a qual propõe que o feto é capaz de se adaptar a um ambiente intra-uterino adverso otimizando o uso de suprimentos energéticos reduzidos, no sentido de garantir sua sobrevivência. Entretanto, esse processo adaptativo levaria ao favorecimento metabólico de órgãos nobres em detrimento de outros, conferindo alterações persistentes no crescimento e função dos teci$\operatorname{dos}^{20}$. Embora constituindo um capítulo importante no estudo das associações entre a vida inicial e o risco para doenças crônicas, a hipótese do fenótipo poupador não explica uma série de achados descritos posteriormente por diferentes grupos, como, por exemplo, as influências da vida fetal sobre a homeostase hídrica ${ }^{21}$, também persistentes, porém sem valor adaptativo imediato. Em outras palavras, a hipótese de Barker não explica acertos metabólicos persistentes que acontecem em resposta a variações do ambiente fetal e que não são imediatamente necessários para a sobrevivência do indivíduo.

No mesmo período, um grupo independente de pesquisadores concentrou-se no estudo dos efeitos da dieta utilizada por RN pré-termo sobre diferentes desfechos tardios. Esses autores demonstraram, através de estudos clínicos, a influência dos diferentes tipos de dieta láctea sobre o crescimento somático, imune e o desenvolvimento neuropsicomotor ${ }^{22,23}$, propondo o uso do termo "programação" nesse contexto. Alinhavado por Dörner et al. ${ }^{24}$, mas amplamente explorado por Alan Lucas, esse termo refere-se ao conceito no qual um 
insulto ou estímulo aplicado em um período crítico ou sensível pode ter efeitos duradouros ou persistentes sobre a estrutura ou função de um organismo ${ }^{25}$. Assim, o desenvolvimento e a gravidade de diversas condições mórbidas dependem da vulnerabilidade genética do indivíduo, da exposição a fatores ambientais adversos, assim como do período de ocorrência desses eventos estressantes ${ }^{26}$. Uma vez que a vida prénatal, a infância e a adolescência são períodos críticos caracterizados por alta plasticidade ${ }^{27,28}$, a exposição a um estímulo significativo pode ter conseqüências de ordem organizacional e produzir alterações persistentes no funcionamento do organismo.

Outro ponto importante diz respeito à constrição materna. Os fetos mamíferos geralmente não atingem seu potencial máximo de crescimento, principalmente porque são afetados por fatores materno-uterinos, como tamanho materno, idade materna, estado nutricional e paridade ${ }^{29}$. Este conceito contribui para a compreensão dos modelos atuais do fenômeno de programação, nos quais se sugere que todo o desenvolvimento fetal é afetado por algum grau de constrição. Em alguns casos, no entanto, a constrição é exacerbada por outros fatores, como doenças materno-placentárias. Ao nascer, essas constrições são aliviadas e as conseqüências se manifestam. O efeito da constrição materna é tão evidente em primíparas que seu impacto tem sido comparado ao do fumo materno ${ }^{30}$. Além disso, a perpetuação do baixo peso através das gerações é explicada em parte por estes mecanismos: a constrição materna será proporcionalmente maior em mães de menor estatura, as quais provavelmente foram RN de baixo peso.

Em diversas espécies animais, influências ambientais atingem o feto através da mãe/placenta ou o neonato através da lactação, promovendo adaptações fisiológicas que aumentam a chance de o indivíduo sobreviver naquele meio. Essas respostas adaptativas preditivas ${ }^{31}$, ao contrário do conceito de fenótipo poupador, não têm um valor adaptativo imediato, mas predizem a adaptação a longo prazo com objetivo de garantir a sobrevivência, no mínimo, até o período reprodutivo. Por exemplo, o estresse materno sinaliza um ambiente externo adverso para o feto, levando a uma hiperreatividade crônica do eixo hipotálamo-hipófise-adrenal (HPA) neste indivíduo, conferindo maior estado de alerta e maiores chances de sobrevivência ${ }^{32,33}$. As respostas adaptativas preditivas causariam uma alteração persistente do funcionamento do organismo, provavelmente através de processos epigenéticos, potencialmente transmissíveis através das gerações. Como aspectos essenciais para a sobrevivência do indivíduo, é de se esperar que a nutrição, o metabolismo, o crescimento, a reprodução e as respostas ao estresse sejam os mais facilmente passíveis de programação.

O modelo das respostas adaptativas preditivas sugere que o organismo em desenvolvimento tem a capacidade de prever o ambiente no qual crescerá, utilizando sinais hormonais maternos através da placenta e/ou através da lactação. Esses sinais fazem o indivíduo ajustar sua fisiologia de acordo com tal inferência. Se a previsão é correta, o risco para doenças é baixo. No entanto, se a inferência for errônea, há um aumento no risco para doenças, que provavelmente irão se manifestar após o período reprodutivo (e, portanto, não há "pressão" da seleção natural contra esta predição errônea durante a evolução). O risco para doenças, então, é o resultado do grau de concordância ou contraste (match or mismatch ${ }^{34}$ ) entre o ambiente previsto pelo indivíduo durante o período de alta plasticidade e desenvolvimento e o ambiente real em que este indivíduo vive na maturidade (Figura 1).

O modelo de concordância ou contraste introduz a idéia de uma plasticidade relacionada ao desenvolvimento (developmental plasticity), fenômeno no qual um genótipo origina uma variedade de estados fisiológicos diversos em resposta a diferentes condições ambientais durante o desenvolvimento. Estudos recentes oferecem suporte a esse modelo através da demonstração de interações entre o ambiente e a expressão gênica em diferentes níveis. Isto é, não apenas o ambiente celular afeta a expressão de genes e a produção protéica, mas as relações do indivíduo com o ambiente também podem influenciar aspectos comportamentais, morfológicos e a expressão gênica, até mesmo em uma questão de horas ${ }^{35}$. Estudos elegantes demonstram que a influência de interações ocorridas em períodos vulneráveis ou passíveis de programação, através de efeitos epigenéticos, podem persistir, inclusive de forma transgeracional ${ }^{36,37}$. Um exemplo importante ilustrando essas teorias em humanos é a presença de um prevalente polimorfismo do gene PPARy e o risco para diabetes, que só é evidente se os indivíduos também têm histórico de menor comprimento ao nascer ${ }^{38}$.

Pelos motivos acima, a epigenética, o estudo de alterações herdadas da expressão gênica que não são devidas à seqüência de nucleotídeos do ADN, tornou-se um modelo fundamental para pesquisa em DOHaD. Seu conceito traz implícito um processo de causalidade importante em nível celular, regulando o crescimento e a diferenciação tecidual, e envolve alterações químicas do ADN (como a metilação) ou de proteínas associadas (como as histonas, que se associam à molécula de ADN nos nucleossomos para formar a cromatina). O padrão de informação epigenética é transmitido através de mitose, sendo específico para determinado tecido e tipo celular e essencial para a manutenção do perfil de expressão gênica do organismo. Como os efeitos epigenéticos podem sofrer interferência do ambiente durante o desenvolvimento, estes são extremamente relevantes no campo de estudos de DOHaD.

\section{O eixo hipotálamo-hipófise-adrenal no início da vida e mecanismos de programação}

A exposição aos glicocorticóides no período fetal tem sido proposta como um dos principais fatores na programação do maior risco para doenças crônicas em indivíduos nascidos com baixo peso ${ }^{39}$, associando-se a uma maior probabilidade de 


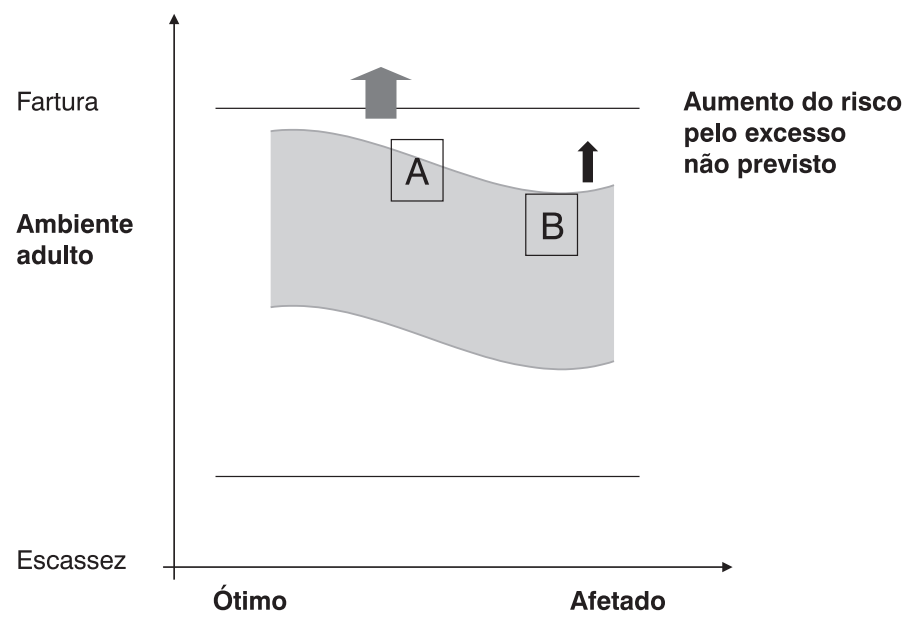

Ambiente durante o desenvolvimento

Figura 1 - Relação entre os ambientes durante o desenvolvimento e na vida adulta. As linhas horizontais representam os limites do ambiente ao qual o indivíduo é exposto; a área acinzentada representa a zona de respostas preditivas adaptativas apropriadas, associada a um risco para doenças reduzido. O indivíduo $A$, exposto a um ambiente intra-uterino adequado, é capaz de tolerar uma variação ambiental maior na vida adulta sem o desenvolvimento de estados mórbidos, ao contrário do indivíduo B (adaptado de Gluckman \& Hanson ${ }^{34}$ )

desenvolvimento tardio de hipertensão, diabetes e distúrbios psiquiátricos, como depressão e ansiedade. A atividade alterada do eixo HPA em períodos vulneráveis do desenvolvimento estaria envolvida na programação do padrão de saúde do indivíduo. Essa alteração seria determinada pela exposição crônica aos glicocorticóides maternos ou ao estresse durante a gestação ${ }^{33}$.

Os glicocorticóides constituem a maior subclasse de hormônios esteróides que regulam processos metabólicos, cardiovasculares, imunitários e comportamentais ${ }^{26,40}$. Seus efeitos fisiológicos são mediados por uma proteína citosólica de 94 kD, o receptor glicocorticóide (GR). O GR está amplamente distribuído pelo cérebro e tecidos periféricos. No estado inativo, o GR é parte de um complexo multiprotéico consistindo de várias moléculas de proteínas de choque térmico ${ }^{41-43}$. Ligando-se aos glicocorticóides, o GR transloca-se para o núcleo da célula, onde interage com elementos responsivos aos glicocorticóides específicos no ADN, de modo a alterar a transcrição de determinados genes ${ }^{44}$. $O$ receptor ativado também inibe, via interações proteínaproteína, outros fatores de transcrição, como o c-jun/c-fos e NF-kB, que são reguladores positivos da transcrição de vários genes envolvidos na ativação e crescimento de células imunes e de outros tipos celulares ${ }^{45}$. Além disso, os glicocorticóides alteram a estabilidade do ARN mensageiro e, portanto, a tradução de várias proteínas, assim como o potencial elétrico de neurônios. Na maioria dos vertebrados, há um ritmo circadiano pronunciado da secreção de glicocorticóides, com picos relacionados ao início da fase ativa do ciclo diurno ${ }^{46}$. O ritmo circadiano glicocorticóide é dependente do núcleo supraquiasmático; lesões dessa estrutura do tronco cerebral levam a um nível aproximadamente constante e intermediário entre o pico e o nadir circadianos ${ }^{47,48}$.

A regulação das ações do eixo HPA é feita, em grande parte, pela retroalimentação negativa dos glicocorticóides sobre componentes do sistema nervoso central (SNC), aumentando ou diminuindo sua atividade de acordo com as necessidades fisiológicas ${ }^{49}$. Várias estruturas cerebrais estão envolvidas nos processos de retroalimentação, dentre as quais se destacam o hipotálamo, a amígdala, o córtex cerebral pré-frontal e o hipocampo ${ }^{50}$, sendo esta última estrutura uma das mais fortemente relacionadas à regulação do eixo, em função de sua alta concentração de receptores glicocorticóides.

Os glicocorticóides têm importância fundamental na gestação em mamíferos, uma vez que estão envolvidos nas adaptações metabólicas maternas ${ }^{51}$. Além disso, atuam na coordenação entre a aptidão para o nascimento e o início dos mecanismos de parto. Durante a gestação, os esteróides lipofílicos facilmente atravessam a placenta, mas os níveis de glicocorticóides fetais são muito menores que os maternos ${ }^{52,53}$. Isso se deve à intensa atuação na placenta da enzima $11 \beta$ hidróxi-esteróide-desidrogenase tipo 2 (11ß-HSD-2), que catalisa a conversão dos glicocorticóides fisiologicamente ativos cortisol e corticosterona em formas inertes como a cortisona ${ }^{54}$. Assim, forma-se uma barreira que protege o feto da exposição aos glicocorticóides maternos, embora ainda permita a passagem de cerca de 10 a $20 \%{ }^{55}$. É interessante notar 
que há uma correlação positiva entre o peso de nascimento e a atividade dessa enzima em ratos ${ }^{56}$ e humanos ${ }^{57}$.

Em humanos, os níveis plasmáticos maternos de hormônio liberador de corticotropina $(\mathrm{CRH}$, produzido pela placenta) aumentam exponencialmente conforme a gestação avança, tendo seu pico no momento do parto. Em partos prematuros, esse aumento é muito mais rápido ${ }^{58}$. O CRH placentário atinge o feto, embora em concentrações menores que na mã $\mathrm{e}^{59}$. No feto, há receptores de $\mathrm{CRH}$ na pituitária ${ }^{60}$ e na adrenal $^{61}$. A estimulação da pituitária fetal pelo CRH aumenta a produção de $\mathrm{ACTH}$ e, conseqüentemente, de cortisol pela adrenal, amadurecendo o eixo HPA fetal e induzindo a formação de surfactante nos pulmões.

A responsividade do eixo HPA em mamíferos flutua no período perinatal, sendo moderadamente responsiva no momento do nascimento, mas diminuindo em intensidade no período neonatal ${ }^{62,63}$. Em ratos, há um pico de corticosterona no último estágio fetal, seguido de pouca responsividade até o fim da segunda semana de vida extra-uterina, fato conhecido como período hiporresponsivo ao estresse ${ }^{64,65}$. Caracteristicamente, há uma exacerbação do mecanismo de retroalimentação negativa dos glicocorticóides na hipófise e diminuição da sensibilidade da adrenal ao ACTH neste período ${ }^{66}$. Há evidências de que este período hiporresponsivo também exista em humanos ${ }^{67,68}$.

Conforme propõe o conceito de programação, a exposição a um estímulo ou estressor nesses primeiros dias determina alterações neuroquímicas e comportamentais observáveis durante toda a vida. Embora "hiporresponsivos", esses indivíduos respondem agudamente ao estresse de separação da mãe mesmo se não expostos a nenhum estressor adicional ${ }^{69}$, sendo que a resposta aumenta progressivamente nas 24 horas subseqüentes. Além disso, nessa fase, os níveis de transcortina são muito baixos, sendo que a maior parte dos glicocorticóides circula no plasma em sua forma não-ligada e, portanto, biologicamente ativa ${ }^{70,71}$. Logo, mesmo que a concentração total de glicocorticóides plasmáticos seja baixa no período hiporresponsivo, a concentração da sua forma biologicamente ativa é relativamente alta, suficiente para que o hormônio exerça suas ações biológicas e possivelmente atue programando o SNC de forma persistente.

Um exemplo interessante de interação entre gene e ambiente, no que concerne o eixo HPA, é o modelo que avalia diferenças naturais do cuidado materno em ratos, mostrando que o meio no qual os filhotes crescem se correlaciona com sua reatividade ao estresse, sendo que filhotes de mães altamente cuidadoras serão menos responsivos em relação a filhotes de mães pouco cuidadoras ${ }^{72}$. Sugere-se que a estimulação tátil de mães altamente cuidadoras atue através de vias serotoninérgicas ascendentes do núcleo da rafe ${ }^{73}$, que induzem a expressão de receptores glicocorticóides no hipocampo $^{74,75}$. A serotonina atua provavelmente através do receptor $5 \mathrm{HT7}$, que é regulado por glicocorticóides ${ }^{76}$ e positivamente ligado ao AMP cíclico ${ }^{77}$. Ocorre, então, a estimulação de fatores de transcrição associados ao AMPc, como o fator de crescimento do nervo induzível pelo fator $A(N G F I-A)^{78}$. Embora a afinidade do NGFI-A ao seu sítio de reconhecimento na seqüência de ADN responsável pela produção de GR seja baixa, a estimulação tátil promove uma grande elevação nos níveis deste fator de transcrição, aumentando, portanto, a chance de ligação ${ }^{78}$. A ligação do NGFI-A resulta em recrutamento de histonas-acetiltransferases, que aumentam a acetilação das histonas, facilitando o acesso de desmetilases e a desmetilação do sítio promotor do $\mathrm{GR}^{79}$. O sítio promotor desmetilado exibirá alta afinidade ao NGFI-A, resultando em uma maior atividade do promotor de GR induzido por NGFI-A no hipocampo, uma maior produção de receptores glicocorticóides nessa estrutura e, portanto, um mecanismo de retroalimentação negativa mais eficiente.

\section{A importância do padrão de crescimento na infância}

Os estudos iniciais relacionando baixo peso ao nascer com maior risco cardiovascular baseavam-se principalmente na restrição de crescimento intra-uterino (RCIU) como o fator causal dessas associações. Evidências recentes também sugerem que o padrão de crescimento da criança durante os primeiros anos de vida seja fortemente influenciado pelo padrão de crescimento fetal, o que pode determinar uma elevação na probabilidade de ocorrência de desfechos metabólicos desfavoráveis. Alterações no padrão de crescimento após o nascimento, principalmente em RN pré-termo com RCIU, relacionam-se significativamente com o desenvolvimento de doenças crônicas em idades subseqüentes.

A conduta corrente recomenda estimular e promover o crescimento do lactente com baixo peso visando a redução das taxas de morbimortalidade e preservação de aspectos neurocognitivos. Entretanto, alguns estudos clínicos propõem que o rápido ganho de peso (catch up) neste período precoce possa estar associado a maior incidência de doença cardiovascular na maturidade ${ }^{80,81}$.

Os riscos para doenças cardiovasculares ${ }^{82}$ e para obesidade $^{83}$ são consistentemente encontrados em grupos de indivíduos com um grande aumento no peso corporal entre o nascimento e a idade escolar ou pré-adolescência, primariamente naqueles que eram pequenos ou que tinham baixo peso ao nascer. Ou seja, as conseqüências de um determinado peso corporal são condicionadas pelo crescimento tanto intraútero quanto durante a infância tardia. Logo, os padrões de crescimento que predispõem ao desenvolvimento de doenças crônicas são complexos, sendo importante fazer a distinção entre catch up precoce e tardio. Enquanto o primeiro parece ser benéfico conforme discutido acima, a obesidade na infância sabidamente tem efeitos deletérios a longo prazo, especialmente nesta população de bebês nascidos com baixo peso, e deve ser prevenida.

Por esses motivos, apesar de ter sido aprovado pelo Food and Drug Administration (FDA) em 2001 e na Europa em 2003, e de atualmente ser recomendado na literatura especializada ${ }^{84}$, o uso de hormônio de crescimento $(\mathrm{GH})$ para 
crianças pequenas para a idade gestacional e com baixa estatura continua um assunto controverso. Por volta de $10 \%$ dos indivíduos pequenos para a idade gestacional não apresentam catch up e se mantêm abaixo do percentil 3 para peso e altura por toda a infância, tendo menor altura na vida adulta. É certo que o aumento na altura final tem seu impacto psicossocial reconhecido, porém o conhecimento que o catch up tardio pode ter conseqüências danosas a longo prazo para esta população torna a indicação de terapia uma decisão árdua. $O$ próprio consenso de manejo de indivíduos nascidos pequenos para a idade gestacional, que recomenda o uso de GH durante toda a infância e adolescência a fim de aumentar a altura final, diz que "atualmente não se sabe se a terapia com GH para sujeitos nascidos pequenos para a idade gestacional durante a infância e adolescência é associada a benefícios ou amplificação dos riscos (como conseqüências metabólicas) na vida adulta"84. Apesar de reconhecerem os estudos epidemiológicos relatando maior risco para doenças cardíacas, acidente vascular cerebral, hipertensão, obesidade e diabetes melito nesta população, os autores argumentam que não há evidências suficientes para fazer qualquer tipo de seguimento especial ou diferencial em relação às outras crianças. De qualquer modo, é importante ressaltar que o crescimento fetal deve ser considerado fator de risco relevante para doenças crônicas, embora não possa ser encarado como um fator causal. Em outras palavras, as doenças crônicas não são programadas por si, mas a tendência para o desenvolvimento das doenças parece ser programada. Há inúmeras interações entre o crescimento fetal e variáveis envolvendo o ambiente na infância e adolescência, portanto a melhor abordagem do problema é o foco na perspectiva do curso da vida e suas interações com a história fetal e neonatal.

\section{Conseqüências a longo prazo da nutrição nos primeiros anos de vida}

A associação entre eventos neonatais e maior risco para doenças na vida adulta não é necessariamente ligada apenas ao baixo peso ao nascer. Neste caso, outros mecanismos e sinais para programação parecem ser importantes. Dentre estes, um dos mais estudados é a nutrição no início da vida, que ainda é pouco conhecida.

A literatura do início do século XX já demonstrava interesse em relação ao impacto da nutrição após o nascimento sobre o crescimento somático e de órgãos específicos através de manipulações dietéticas em períodos críticos ${ }^{85,86}$. Atualmente e principalmente devido aos estudos de DOHaD, existem sugestões de que a nutrição em períodos vulneráveis possa alterar a estrutura da cromatina e a expressão gênica, assim como potencialmente influenciar a saúde do indivíduo a longo prazo ${ }^{87}$.

Sabe-se que crianças que recebem leite materno apresentam uma cinética de crescimento diferente de crianças que recebem fórmula ${ }^{88}$. A literatura propõe que o RN em uso do leite materno receba uma quantidade calórica suficiente para crescer, mas não superior à necessária, além de ganhar peso de forma mais lenta que um RN alimentado com fórmula artificial. Como estudos experimentais demonstram que o excesso alimentar no período neonatal associa-se a maior risco para obesidade e síndrome metabólica na vida adulta ${ }^{89}$, é possível que este seja um dos mecanismos pelos quais o aleitamento materno possa proteger contra doenças ao longo da vida. Sabe-se também que as diferenças constitucionais entre o leite materno e a fórmula artificial, como a quantidade de calorias e proteínas, é uma variável importante neste contexto.

Outro possível fator envolvido é a diferença no comportamento alimentar de bebês que recebem aleitamento materno, sendo que estes RN apresentam uma freqüência de sucção diferenciada ${ }^{90}$, assim como demonstram maior grau de controle da quantidade de leite ingerida ${ }^{91}$. Além disso, a variação constante no sabor e odor do leite materno expõe o RN à experiência de diferentes estímulos gustatórios, o que favorece o consumo de alimentos com sabor semelhante mais tarde na vida92, ampliando a variedade alimentar do indivíduo.

Especificamente em relação à proteção ao longo da vida, mostrou-se, por exemplo, que o aleitamento materno em RN pré-termo se associa a um melhor perfil lipídico plasmático na adolescência ${ }^{92}$, assim como a menores níveis pressóri$\cos ^{93}$. Entretanto, tem sido mais difícil provar com clareza o efeito da dieta pós-natal sobre a composição corporal do adulto. Embora vários estudos incluindo meta-análises tenham demonstrado que o aleitamento materno está associado a menor incidência de obesidade usando o índice de massa corporal (IMC) como variável categórica ${ }^{94,95}$, os estudos que se concentram em composição corporal não obtiveram sucesso em estabelecer esta associação, ou encontraram apenas efeitos muito discretos ${ }^{96}$. A mesma falta de associação também foi descrita no Brasil ${ }^{97}$. Conforme ressaltado por Wells et al. ${ }^{98}$, várias dificuldades metodológicas impedem uma melhor avaliação dos efeitos do aleitamento materno, incluindo a falta de informações precisas nos grandes estudos de coorte, assim como a influência de fatores sociais e a impossibilidade de realização de estudos randomizados. Em adição, este autor menciona também a possibilidade de causalidade reversa, ou seja, que as dificuldades durante o aleitamento materno possam levar ao emprego de uma dieta agressiva e ao excesso alimentar em RN alimentados com fórmula, aumentando a sua chance de desenvolverem alterações no padrão de saúde na vida adulta quando comparados a bebês que receberam aleitamento materno.

Embora alguns estudos apontem para os benefícios agudos da suplementação alimentar com ácidos graxos poliinsaturados de cadeia longa ( $n-3$ e n-6 LCPUFA) nas fórmulas artificiais $^{99}$, assim como de pré e probióticos para a mãe durante a gestação e aleitamento, seus efeitos em longo prazo no desenvolvimento neurológico e imunológico dos indivíduos ainda não são claros, sendo que mais estudos são necessários neste contexto. 


\section{Métodos de abordagem científica}

O DOHaD é uma linha de pesquisa que agrega informações de diferentes áreas do conhecimento, sendo uma interface interessante entre as ciências biológicas e a área da saúde. As questões e hipóteses apresentam diferentes abordagens científicas, que incluem:

- Estudos epidemiológicos. Grandes estudos de coorte baseados em bancos de dados perinatais elaborados no início do século $X X$ foram a base para a formação dos primeiros modelos de $\mathrm{DOH}$ D, principalmente através dos estudos de Barker et al. Embora seja a abordagem mais apropriada para ressaltar associações entre eventos precoces e desfechos tardios, os estudos epidemiológicos não provam causalidade. Além disso, coortes em humanos são dispendiosas e exigem grande envolvimento da equipe de pesquisadores e também dos indivíduos estudados, trazendo resultados apenas a longo prazo quando se consideram estudos de $\mathrm{DOHaD}$. A perda do seguimento durante $\mathrm{o}$ período do estudo, o questionamento sobre a validade das associações encontradas em adultos para a população pediátrica atual e sobre a transposição dos achados entre as diferentes populações mundiais limitam as aplicações dos resultados.

- Estudos clínicos. A comparação de indivíduos com histórias perinatais diversas em suas características clínicas, comportamento e/ou resposta a intervenções é o objetivo de estudos clínicos de DOHaD. De realização relativamente mais rápida do que estudos de coorte, porém ainda limitados por questões éticas, os estudos clínicos têm esclarecido questões importantes da área.

- Estudos experimentais. De relativa facilidade de execução, menos onerosos, mais rápidos e altamente informativos, estudos realizados em animais contribuem com uma grande facção de informações existentes na área. No entanto, a extrapolação de resultados para humanos é extremamente delicada e frágil. Por exemplo, muitas das espécies utilizadas têm gestações múltiplas, o que dificulta a comparação com humanos. Espécies com gestações únicas têm sido propostas como alternativas (ovinos, por exemplo), porém a diferença entre o grau de maturidade dos filhotes ao nascer também interfere nas comparações.

- Estudos translacionais. Este conceito de pesquisa científica, em que o pesquisador se divide "entre a bancada e o leito", tem um importante número de adeptos entre os estudiosos de DOHaD. Com a facilidade de realização de estudos de área básica, pesquisadores aplicam os conhecimentos adquiridos para gerar hipóteses e questões relevantes à fisiologia humana, adaptando os protocolos para testes factíveis em pacientes. Reunindo as vantagens de estudos epidemiológicos/clínicos com as dos estudos básicos, esta nova tendência traz um grande volume de informações relevantes e tem contribuído de maneira sólida para a construção de conhecimento em DOHaD.

\section{DOHaD em países em desenvolvimento}

A maioria dos estudos de DOHaD tem sido produzida em países desenvolvidos, e a relevância da aplicação dessas evidências para a saúde de países em desenvolvimento só agora começa a ser estabelecida. O modelo da concordância ou contraste, em que o maior risco para doenças crônicas é o resultado do grau de contraste entre o ambiente fetal e o meio onde o indivíduo cresce, reflete a experiência de muitas pessoas que vivem em países em desenvolvimento. A incidência de RCIU é alta nesses locais, em parte pela alta prevalência de desnutrição entre as mulheres. Por outro lado, a ascensão econômica e melhoramentos na agricultura têm transformado a nutrição de crianças e adultos, e a emergência de obesidade nos países em desenvolvimento tem sido relatada em diferentes locais do mundo. O Brasil também segue este padrão de contraste, mesmo quando diferentes classes sociais são consideradas ${ }^{100}$.

É importante notar que, nas grandes coortes da Europa que deram início aos estudos de DOHaD (Hertfordshire, Inglaterra e Helsinque, Finlândia), a associação entre baixo peso ao nascer e doenças cardiovasculares é significativa, mesmo quando não se considera o IMC atual dos indivíduos na análise. No entanto, estudos semelhantes feitos na África ${ }^{101}$, China ${ }^{102}$ e Índia ${ }^{103}$ só são capazes de encontrar a mesma associação significativa quando ajustam a análise para o IMC adulto. Isso sugere um padrão diferente nos países em desenvolvimento, nos quais ter nascido com baixo peso se torna um problema apenas quando acompanhado de obesidade.

Um interessante estudo feito na Índia ${ }^{104}$ mostra que adultos resistentes à insulina nascidos com baixo peso eram caracterizados por um aumento do IMC entre as idades de 2 e 12 anos, embora não atingissem níveis considerados como sobrepeso ou obesidade pelos padrões internacionais. Portanto, é possível que a simples recuperação do peso após um período de desnutrição inicial seja um parâmetro mais importante do que a obesidade em termos absolutos nas populações dos países em desenvolvimento.

O conceito de DOHaD tem enorme potencial de implicações para estratégias de saúde pública nesses países, e isso foi inclusive reconhecido pela Organização Mundial da Saúde (OMS) em 2004. Entretanto, apesar da urgência suscitada pela epidemia de doenças crônicas nesses locais, esta é ainda uma área de conhecimento pouco explorada, não sendo possível propor qualquer recomendação clara no nível de saúde pública na situação corrente.

\section{Aspectos éticos}

Os avanços na medicina fetal e neonatal têm sido acompanhados por uma mudança na visão do status fetal. A emergência de "fetos como pacientes" traz pressão moral tanto para a equipe médica quanto para a futura mãe. O conceito de "direitos fetais" - "direito à vida", "direito a nascer"- é agora estendido para "direito a nascer saudável". De uma maneira mais ou menos extremista, o feto e a mãe têm sido 
encarados como duas entidades ética e legalmente independentes, e os aspectos éticos envolvendo algumas circunstâncias de conflito entre essas duas entidades devem ser cuidadosamente avaliados.

Não é raro ouvirmos discursos descuidados visando a promoção da saúde pública, como os que insinuam que gestantes necessitam "vigilância constante para promover a saúde e o bem-estar fetal". Além disso, a associação entre o peso ao nascer e as doenças crônicas pode resultar em uma enorme sobrecarga de responsabilidades para a mãe. Assim, o feto tem não apenas o "direito a nascer saudável", mas sim o "direito a nascer tendo uma vida adulta saudável".

A mensagem-chave dos conceitos de DOHaD é que aspectos relacionados ao ambiente precoce são fundamentais no desenvolvimento de doenças, e não que a biologia do indivíduo torna a doença inevitável. Porém, a ênfase excessiva no crescimento fetal e em aspectos relacionados à gestação facilita o surgimento de julgamentos precipitados, "acusando" as mães de não saberem fazer escolhas para seu estilo de vida e afetarem persistentemente a saúde de seus filhos.

Ainda é importante mencionar estudos que relacionam eventos biológicos com influências sobre o comportamento do indivíduo. Assim, conceitos como "programação" tomam uma dimensão determinista e questionam a capacidade dos indivíduos afetados de fazerem as escolhas apropriadas para a sua própria saúde, excluindo importantes variáveis, como fatores sociais e econômicos. A "sentença" de ter nascido com baixo peso e ser "condenado" a um determinado futuro é uma abordagem questionável. Mais relevante do que isso é a busca pela compreensão dos mecanismos que levam a essas associações, principalmente considerando influências sociais, a fim de auxiliar no desenvolvimento de medidas preventivas.

\section{Conclusões}

Em seu artigo de 2007, Gluckman \& Hanson propõem direções futuras para a pesquisa na área de $\mathrm{DOHaD}$, as quais revisaremos aqui ${ }^{102}$. Embora 20 anos tenham passado desde a publicação das primeiras observações relacionando o ambiente precoce com risco para o desenvolvimento de doenças crônicas, há ainda uma série de questões a serem respondidas. Um debate importante relaciona-se ao impacto desses processos na prevalência das doenças crônicas. Atualmente, os estudos têm enfatizado relações entre variáveis da vida perinatal e desfechos específicos, como a resistência à insulina ou alterações associadas a doenças cardiovasculares (hipertensão, obesidade e hipercolesterolemia, por exemplo). No entanto, a contribuição da influência de variáveis precoces para a gênese do fenótipo metabólico deveria ser estudada mais apropriadamente.

A definição de "contraste" também necessita ser estabelecida de forma mais robusta, assim como quais parâmetros são marcadores de contraste. O catch up, apesar de extensamente utilizado em estudos, também merece maior precisão: os estudos devem se concentrar em detalhar de qual tipo de catch up (em peso, em massa magra, etc.) estão tratando.

O DOHaD deu origem a uma série de estudos voltados a investigar a relação entre variações ambientais na vida precoce, polimorfismos em genes relacionados e desfechos metabólicos. Porém, também resta saber até que ponto é possível estabelecer especificidades dessas variações para uma população determinada e como esses polimorfismos interagem com as dicas ambientais, influenciando o risco para o desenvolvimento de doenças crônicas. Do mesmo modo, é irreal imaginar que um único gene possa ser responsável por tamanha miríade de desfechos. Uma visão ampla integrando variáveis genéticas, epigenéticas e ambientais é necessária. Os estudos ainda buscarão aprofundar investigações sobre as diferentes classes de genes-alvo e seus papéis nestes processos, como, por exemplo, genes regulatórios, genes relacionados ao crescimento e genes mitocondriais. A sensibilidade tecidual específica para hormônios e outras moléculas sinalizadoras, que possivelmente flutua durante o desenvolvimento, deve ser caracterizada em diferentes fases da vida. Além disso, situações de herança não-genômica, como o exemplo da constrição materna ressaltado acima, devem ser ainda melhor descritas.

É importante salientar que muitas outras características humanas podem sofrer influências de eventos traumáticos ocorridos precocemente, tais como o comportamento, a reprodução, a homeostase térmica e hídrica e as funções cognitivas. Interessante notar que, nesses casos, essas alterações podem apresentar um fenótipo metabólico alterado. A questão mais importante aqui é a possibilidade de existência de uma ponte causal entre indução de fenótipos específicos em domínios diversos e dicas ambientais comuns na vida precoce.

Por fim, relativamente poucos estudos até hoje dedicaram-se a investigar os aspectos de intervenção e reversão de fenótipo. Estudos em animais utilizando suplementações dietéticas têm demonstrado resultados interessantes, porém a aplicabilidade destes protocolos para humanos é delicada. São necessários mais estudos de área básica dando suporte para intervenções clínicas.

\section{Agradecimentos}

Os autores agradecem a importante contribuição dos professores Danilo Blank, Humberto Rosa e Gilberto Eckert na revisão dos originais e à professora Sabrina Abreu, pela importante contribuição em relação às definições terminológicas.

\section{Referências}

1. Avery ME, Mead J. Surface properties in relation to atelectasis and hyaline membrane disease. AMA J Dis Child. 1959;97:51723.

2. De Souza Campos Filho P, Montenegro MR, De Almeida AD. Necrotizing enterocolitis (intestinal gangrene). Rev Bras Gastroenterol. 1952;4:929-34. 
3. Berdon WE, Grossman H, Baker DH, Mizrahi A, Barlow O, Blanc WA. Necrotizing enterocolitis in the premature infant. Radiology. 1964;83:879-87.

4. Northway WH Jr., Rosan RC, Porter DY. Pulmonary disease following respirator therapy of hyaline-membrane disease. Bronchopulmonary dysplasia. N Engl J Med. 1967;276:357-68.

5. Terry TL. Fibroblastic overgrowth of persistent tunica vasculosa lentis in infants born prematurely: II. Report of cases-clinical aspects. Trans Am Ophthalmol Soc. 1942;40:262-84.

6. Kermack WO, McKendrick AG, McKinlay PL. Death-rates in Great Britain and Sweden. Some general regularities and their significance. Lancet. 1934:698-703.

7. Ravelli GP, Stein ZA, Susser MW. Obesity in young men after famine exposure in utero and early infancy. N Engl J Med. 1976;295:349-53.

8. Neel JV. Diabetes mellitus: a "thrifty" genotype rendered detrimental by "progress"? Am J Hum Genet. 1962;14:353-62.

9. Barker DJ, Winter PD, Osmond C, Margetts B, Simmonds SJ. Weight in infancy and death from ischaemic heart disease. Lancet. 1989;2:577-80.

10. Barker DJ, Eriksson JG, Forsen T, Osmond C. Fetal origins of adult disease: strength of effects and biological basis. Int J Epidemiol. 2002;31:1235-9.

11. Eriksson JG, Forsen T, Tuomilehto J, Jaddoe VW, Osmond C, Barker DJ. Effects of size at birth and childhood growth on the insulin resistance syndrome in elderly individuals. Diabetologia. 2002;45:342-8.

12. Davies AA, Smith GD, Ben-Shlomo Y, Litchfield P. Low birth weight is associated with higher adult total cholesterol concentration in men: findings from an occupational cohort of 25,843 employees. Circulation. 2004;110:1258-62.

13. Szathmari M, Vasarhelyi B, Szabo M, Szabo A, Reusz GS, Tulassay $T$. Higher osteocalcin levels and cross-links excretion in young men born with low birth weight. Calcif Tissue Int. 2000;67:429-33.

14. Jones A, Godfrey KM, Wood P, Osmond C, Goulden P, Phillips DI. Fetal growth and the adrenocortical response to psychological stress. J Clin Endocrinol Metab. 2006;91:1868-71.

15. Painter RC, de Rooij SR, Bossuyt PM, de Groot E, Stok WJ, Osmond $\mathrm{C}$, et al. Maternal nutrition during gestation and carotid arterial compliance in the adult offspring: the dutch famine birth cohort. J Hypertens. 2007; 25:533-40.

16. Jones A, Beda A, Ward AM, Osmond C, Phillips DI, Moore VM, et al. Size at birth and autonomic function during psychological stress. Hypertension. 2007;49:548-55.

17. de Rooij SR, Painter RC, Phillips DI, Osmond C, Michels RP, Godsland IF, et al. Impaired insulin secretion after prenatal exposure to the Dutch famine. Diabetes Care. 2006;29:1897901.

18. Rice F, Harold GT, Thapar A. The effect of birth-weight with genetic susceptibility on depressive symptoms in childhood and adolescence. Eur Child Adolesc Psychiatry. 2006;15:383-91.

19. Thompson C, Syddall H, Rodin I, Osmond C, Barker DJ. Birth weight and the risk of depressive disorder in late life. $\mathrm{Br}$ J Psychiatry. 2001;179:450-5.

20. Hales CN, Barker DJ. Type 2 (non-insulin-dependent) diabetes mellitus: the thrifty phenotype hypothesis. Diabetologia. 1992;35:595-601.

21. El-Haddad MA, Desai M, Gayle D, Ross MG. In utero development of fetal thirst and appetite: potential for programming. J Soc Gynecol Investig. 2004;11:123-30.
22. Lucas A, Brooke OG, Morley R, Cole TJ, Bamford MF. Early diet of preterm infants and development of allergic or atopic disease: randomised prospective study. BMJ. 1990;300:837-40.

23. Lucas A, Morley R, Cole TJ. Randomised trial of early diet in preterm babies and later intelligence quotient. BMJ. 1998; 317:1481-7.

24. Dörner G. Perinatal hormone levels and brain organization. In: Anatomical neuroendocrinology. Stumpf WE, Grant LD, editors. Basel: S. Karger; 1975. p. 245-52.

25. Lucas A. Programming by early nutrition in man. In: The childhood environment and adult disease. Bock GR, Whelan J, editors. CIBA Foundation Symposium 156. Chichester, UK: Wiley; 1991. p. 38-55.

26. Charmandari E, Tsigos C, Chrousos G. Endocrinology of the stress response. Annu Rev Physiol. 2005;67:259-84.

27. Khazipov R, Luhmann HJ. Early patterns of electrical activity in the developing cerebral cortex of humans and rodents. Trends Neurosci. 2006;29:414-8.

28. Crews F, He J, Hodge C. Adolescent cortical development: a critical period of vulnerability for addiction. Pharmacol Biochem Behav. 2007;86:189-99.

29. Ounsted M, Ounsted C. On fetal growth rate: its variations and their consequences. London: Heinemann Medical; 1973.

30. Ong KK, Preece MA, Emmett PM, Ahmed ML, Dunger DB; ALSPAC Study Team. Size at birth and early childhood growth in relation to maternal smoking, parity and infant breast-feeding: longitudinal birth cohort study and analysis. Pediatr Res. 2002;52:863-7.

31. Gluckman PD, Hanson MA. Developmental origins of disease paradigm: a mechanistic and evolutionary perspective. Pediatr Res. 2004;56:311-7.

32. Van den Bergh BR, Mulder EJ, Mennes M, Glover V. Antenatal maternal anxiety and stress and the neurobehavioural development of the fetus and child: links and possible mechanisms. Neurosci Biobehav Rev. 2005;29:237-58.

33. Weinstock M. The potential influence of maternal stress hormones on development and mental health of the offspring. Brain Behav Immun. 2005; 19:296-308.

34. Gluckman PD, Hanson MA. Living with the past: evolution, development, and patterns of disease. Science. 2004; 305:1733-6.

35. Parikh VN, Clement T, Fernald RD. Physiological consequences of social descent: studies in Astatotilapia burtoni. J Endocrinol. 2006;190:183-90

36. Champagne F, Meaney MJ. Like mother, like daughter: evidence for non-genomic transmission of parental behavior and stress responsivity. Prog Brain Res. 2001;133:287-302.

37. Zhang TY, Bagot R, Parent C, Nesbitt C, Bredy TW, Caldji C, et al. Maternal programming of defensive responses through sustained effects on gene expression. Biol Psychol. 2006;73:7289.

38. Eriksson JG, Lindi V, Uusitupa M, Forsen TJ, Laakso M, Osmond C, et al. The effects of the Pro12Ala polymorphism of the peroxisome proliferator-activated receptor-gamma2 gene on insulin sensitivity and insulin metabolism interact with size at birth. Diabetes. 2002;51:2321-4.

39. Seckl JR, Meaney MJ. Glucocorticoid programming. Ann N Y Acad Sci. 2004;1032:63-84.

40. Sapolsky RM, Romero LM, Munck AU. How do glucocorticoids influence stress responses? Integrating permissive, suppressive, stimulatory, and preparative actions. Endocr Rev. 2000;21:5589. 
41. Bamberger CM, Schulte HM, Chrousos GP. Molecular determinants of glucocorticoid receptor function and tissue sensitivity to glucocorticoids. Endocr Rev. 1996;17:245-61.

42. Giguere V, Hollenberg SM, Rosenfeld MG, Evans RM. Functional domains of the human glucocorticoid receptor. Cell. 1986; 46:645-52.

43. Cadepond F, Schweizer-Groyer G, Segard-Maurel I, Jibard N, Hollenberg SM, Giguere V, et al. Heat shock protein 90 as a critical factor in maintaining glucocorticosteroid receptor in a nonfunctional state. J Biol Chem. 1991;266:5834-41.

44. Pratt WB. Glucocorticoid receptor structure and the initial events in signal transduction. Prog Clin Biol Res. 1990;322:119-32.

45. Scheinman RI, Cogswell PC, Lofquist AK, Baldwin AS Jr. Role of transcriptional activation of I kappa B alpha in mediation of immunosuppression by glucocorticoids. Science. 1995; 270:283-6.

46. Keller-Wood M, Dallman MF. Corticosteroid inhibition of ACTH secretion. Endocr Rev. 1984;5:1-24.

47. Cascio CS, Shinsako J, Dallman MF. The suprachiasmatic nuclei stimulate evening ACTH secretion in the rat. Brain Res. 1987; 423:173-8.

48. Moore RY, Eichler VB. Loss of a circadian adrenal corticosterone rhythm following suprachiasmatic lesions in the rat. Brain Res. 1972;42:201-6.

49. Marti O, Andres R, Armario A. Defective ACTH response to stress in previously stressed rats: dependence on glucocorticoid status. Am J Physiol. 1999;277:R869-77.

50. Campeau S, Day HE, Helmreich DL, Kollack-Walker S, Watson SJ. Principles of psychoneuroendocrinology. Psychiatr Clin North Am. 1998;21:259-76.

51. Atkinson HC, Waddell BJ. The hypothalamic-pituitary-adrenal axis in rat pregnancy and lactation: circadian variation and interrelationship of plasma adrenocorticotropin and corticosterone. Endocrinology. 1995;136:512-20.

52. Beitins IZ, Bayard F, Ances IG, Kowarski A, Migeon CJ. The metabolic clearance rate, blood production, interconversion and transplacental passage of cortisol and cortisone in pregnancy near term. Pediatr Res. 1973;7:509-19.

53. Klemcke HG. Placental metabolism of cortisol at mid- and late gestation in swine. Biol Reprod. 1995;53:1293-301.

54. White PC, Mune T, Agarwal AK. 11beta-Hydroxysteroid dehydrogenase and the syndrome of apparent mineralocorticoid excess. Endocr Rev. 1997;18:135-56.

55. Venihaki MA, Carrigan P, Dikkes P, Majzoub JA. Circadian rise in maternal glucocorticoid prevents pulmonary dysplasia in fetal mice with adrenal insufficiency. Proc Natl Acad Sci USA. 2000; 97:7336-41.

56. Benediktsson R, Lindsay RS, Noble J, Seckl JR, Edwards C. Glucocorticoid exposure in utero: a new model for adult hypertension. Lancet. 1993;341:339-41.

57. Stewart PM, Rogerson FM, Mason JI. Type 2 11ß-hydroxysteroid dehydrogenase messenger ribonucleic acid and activity in human placenta and fetal membranes: its relationship to birth weight and putative role in fetal adrenal steroidogenesis. J Clin Endocrinol Metab. 1995; 80:885-90.

58. McLean M, Bisits A, Davies J, Woods R, Lowry P, Smith R. A placental clock controlling the length of human pregnancy. Nat Med. 1995; 1:460-3.

59. Nodwell A, Carmichael L, Fraser M, Challis J, Richardson B. Placental release of corticotrophin-releasing hormone across the umbilical circulation of the human newborn. Placenta. 1999; 20:197-202.
60. Asa SL, Kovacs K, Singer W. Human fetal adenohypophysis: morphologic and functional analysis in vitro. Neuroendocrinology. 1991;53:562-72.

61. Smith R, Mesiano S, Chan EC, Brown S, Jaffe RB. Corticotropinreleasing hormone directly and preferentially stimulates dehydroepiandrosterone sulfate secretion by human fetal adrenal cortical cells. J Clin Endocrinol Metab. 1998;83: 2916-20.

62. Rokicki W, Forest MG, Loras B, Bonnet H, Bertrand J. Free cortisol of human plasma in the first three months of life. Biol Neonate. 1990;57:21-9.

63. Bergant AM, Kirchler H, Heim K, Daxenbichler G, Herold M, Schrocksnadel $\mathrm{H}$. Childbirth as a biological model for stress? Associations with endocrine and obstetric factors. Gynecol Obstet Inv. 1998;45:181-5.

64. Sapolsky RM, Meaney MJ. Maturation of the adrenocortical stress response: neuroendocrine control mechanisms and the stress hyporesponsive period. Brain Res Rev. 1986;11:65-76.

65. Guillet R, Michaelson SM. Corticotropin responsiveness in the neonatal rat. Neuroendocrinology. 1978;27:119-25.

66. Yoshimura S, Sakamoto S, Kudo H, Sassa S, Kumai A, Okamoto R. Sex-differences in adrenocortical responsiveness during development in rats. Steroids. 2003;68:439-45.

67. Gunnar MR, Broderson L, Krueger K, Rigatuso J. Dampening of adrenocortical responses during infancy: normative changes and individual differences. Child Dev. 1996;67:877-89.

68. Larson M, White BP, Cochran A, Donzella B, Gunnar MR. Dampening of the cortisol response to handling at 3-months in human infants and its relation to sleep, circadian cortisol activity, and behavioral distress. Dev Psychobiol. 1998;33:327-37.

69. Kuhn CM, Pauk J, Schanberg SM. Endocrine responses to mother-infant separation in developing rats. Dev Psychobiol. $1990 ; 23: 395-410$.

70. Henning SJ. Plasma concentrations of total and free corticosterone during development in the rat. Am J Physiol. 1978;235:E451-6.

71. Hadjian AJ, Chedin M, Cochet C, Chambaz EM. Cortisol binding to proteins in plasma in the human neonate and infant. Pediatr Res. 1975;9:40-5.

72. Liu D, Diorio J, Tannenbaum B, Caldji C, Francis D, Freedman A, et al. Maternal care, hippocampal glucocorticoid receptors and hypothalamic-pituitary-adrenal responses to stress. Science. 1997;277:1659-62.

73. Smythe JW, Rowe WB, Meaney MJ. Neonatal handling alters serotonin (5-HT) turnover and $5-\mathrm{HT} 2$ receptor binding in selected brain regions: relationship to the handling effect on glucocorticoid receptor expression. Dev Brain Res. 1994; 80:183-9.

74. Mitchell JB, Rowe W, Boksa P, Meaney MJ. Serotonin regulates type II corticosteroid receptor binding in hippocampal cell cultures. J Neurosci. 1990;10:1745-52.

75. Yau JL, Noble J, Seckl JR. Site-specific regulation of corticosteroid and serotonin receptor subtype gene expression in the rat hippocampus following methylenedioxymethamphetamine: role of corticosterone and serotonin. Neuroscience. 1997;78:111-21.

76. Yau JL, Noble J, Widdowson J, SeckI JR. Impact of adrenalectomy on 5-HT6 and 5-HT7 receptor gene expression in the rat hippocampus. Mol Brain Res. 1997;45:182-6.

77. Meaney MJ, Diorio J, Francis D, Weaver S, Yau J, Chapman K, Seckl JR. Postnatal handling increases the expression of cAMPinducible transcription factors in the rat hippocampus: the effects of thyroid hormones and serotonin. J Neurosci. 2000;20:392635. 
78. Encio IJ, Detera-Wadleigh SD. The genomic structure of the human glucocorticoid receptor. J Biol Chem. 1991;266:7182-8.

79. Carvin CD, Parr RD, Kladde MP. Site-selective in vivo targeting of cytosine 5 DNA methylation by zinc-finger proteins. Nucleic Acids Res. 2003;31:6493-501.

80. Singhal A, Cole TJ, Fewtrell M, Deanfield J, Lucas A. Is slower early growth beneficial for long-term cardiovascular health? Circulation. 2004;109:1108-13.

81. Singhal A, Cole TJ, Fewtrell M, Kennedy K, Stephenson T, Elias-Jones $A$, et al. Promotion of faster weight gain in infants born small for gestational age: is there an adverse effect on later blood pressure? Circulation. 2007;115:213-20.

82. Eriksson JG, Forsen T, Tuomilehto J, Osmond C, Barker DJ. Early growth and coronary heart disease in later life: longitudinal study. BMJ. 2001;322:949-53.

83. Bettiol H, Sabbag Filho D, Silva AA, Portella AK, Silveira PP, Goldani $M Z$, et al. Do intrauterine growth restriction and overweight at school age increase the risk for elevated body mass index in young adults? Braz J Med Biol Res. In press 2007.

84. Clayton PE, Cianfarani S, Czernichow P, Johannsson G, Rapaport $R$, Rogol A. Management of the child born small for gestational age through to adulthood: a consensus statement of the International Societies of Pediatric Endocrinology and the Growth Hormone Research Society. J Clin Endocrinol Metab. 2007; 92:804-10.

85. Jackson CM. Structural changes when growth is supressed by undernourishment in albino rats. Am J Anat. 1932;51:347-379.

86. Rudin D. Effects of inanition upon the growth of the brain of the albino rat. The Anat Rec. 1935;62:175-82.

87. Mathers JC. Early nutrition: impact on epigenetics. Forum Nutr. 2007;60:42-8.

88. Kramer MS, Guo T, Platt RW, Vanilovich I, Sevkovskaya Z, Dzikovich I, et al; Promotion of Breastfeeding Intervention Trials Study Group. Feeding effects on growth during infancy. J Pediatr. 2004; 145:600-5.

89. Boullu-Ciocca S, Dutour A, Guillaume V, Achard V, Oliver C, Grino M. Postnatal diet-induced obesity in rats upregulates systemic and adipose tissue glucocorticoid metabolism during development and in adulthood: its relationship with the metabolic syndrome. Diabetes. 2005;54:197-203.

90. Singhal A, Cole TJ, Fewtrell M, Lucas A. Breastmilk feeding and lipoprotein profile in adolescents born preterm: follow-up of a prospective randomised study. Lancet. 2004;363:1571-8.

91. Singhal A, Cole TJ, Lucas A. Early nutrition in preterm infants and later blood pressure: two cohorts after randomised trials. Lancet. 2001;357:413-9.

92. Arenz S, Ruckerl R, Koletzko B, von Kries R. Breast-feeding and childhood obesity--a systematic review. Int J Obes Relat Metab Disord. 2004;28:1247-56.
93. Owen CG, Martin RM, Whincup PH, Smith GD, Cook DG. Effect of infant feeding on the risk of obesity across the life course: a quantitative review of published evidence. Pediatrics. 2005; 115:1367-77.

94. Toschke AM, Martin RM, von Kries R, Wells J, Smith GD, Ness AR. Infant feeding method and obesity: body mass index and dual-energy X-ray absorptiometry measurements at 9-10 y of age from the Avon Longitudinal Study of Parents and Children (ALSPAC). Am J Clin Nutr. 2007;85:1578-85.

95. Victora CG, Barros F, Lima RC, Horta BL, Wells J. Anthropometry and body composition of 18 year old men according to duration of breast feeding: birth cohort study from Brazil. BMJ. 2003; 327:901.

96. Wells JC, Chomtho S, Fewtrell MS. Programming of body composition by early growth and nutrition. Proc Nutr Soc. 2007;66:423-34.

97. Bosma JF, Hepburn LG, Josell SD, Baker K. Ultrasound demonstration of tongue motions during suckle feeding. Dev Med Child Neurol. 1990;32:223-9.

98. Sievers E, Oldigs HD, Santer R, Schaub J. Feeding patterns in breast-fed and formula-fed infants. Ann Nutr Metab. 2002; 46:243-8

99. Mennella JA, Griffin CE, Beauchamp GK. Flavor programming during infancy. Pediatrics. 2004;113:840-5.

100. Silveira PP, Portella AK, Goldani MZ. Obesity in Latin America: similarity in the inequalities. Lancet. 2005;366:451-2.

101. Levitt NS, Lambert EV, Woods D, Hales CN, Andrew R, Seckl JR. Impaired glucose tolerance and elevated blood pressure in low birth weight, nonobese, young south african adults: early programming of cortisol axis. J Clin Endocrinol Metab. 2000;85:4611-8.

102. Zhao M, Shu XO, Jin F, Yang G, Li HL, Liu DK, et al. Birthweight, childhood growth and hypertension in adulthood. Int J Epidemiol. 2002;31:1043-51.

103. Bhargava SK, Sachdev HS, Fall CH, Osmond C, Lakshmy R, Barker DJ, et al. Relation of serial changes in childhood bodymass index to impaired glucose tolerance in young adulthood. N Engl J Med. 2004;350:865-75.

104. Gluckman PD, Hanson MA. Developmentalplasticity and human disease: research directions. J Intern Med. 2007;261:461-71.

Correspondência:

Marcelo Zubaran Goldani

Departamento de Pediatria

Rua Ramiro Barcelos, 2350, Largo Eduardo Zaccaro Faraco

CEP 90035-003 - Porto Alegre, RS

Tel.: (51) 2101.8515

Fax: (51) 2101.8001

E-mail: mgoldani@hcpa.ufrgs.br 